Review

\title{
Folding by Numbers: Primary Sequence Statistics and Their Use in Studying Protein Folding
}

\author{
Brent Wathen and Zongchao Jia * \\ Department of Biochemistry, Queen's University, Kingston, Ontario, Canada K7L 3N6 \\ * Author to whom correspondence should be addressed; E-Mail: jia@queensu.ca
}

Received: 30 January 2009; in revised form: 30 March 2009 / Accepted: 2 April 2009 /

Published: 8 April 2009

\begin{abstract}
The exponential growth over the past several decades in the quantity of both primary sequence data available and the number of protein structures determined has provided a wealth of information describing the relationship between protein primary sequence and tertiary structure. This growing repository of data has served as a prime source for statistical analysis, where underlying relationships between patterns of amino acids and protein structure can be uncovered. Here, we survey the main statistical approaches that have been used for identifying patterns within protein sequences, and discuss sequence pattern research as it relates to both secondary and tertiary protein structure. Limitations to statistical analyses are discussed, and a context for their role within the field of protein folding is given. We conclude by describing a novel statistical study of residue patterning in $\beta$-strands, which finds that hydrophobic $(i, i+2)$ pairing in $\beta$ strands occurs more often than expected at locations near strand termini. Interpretations involving $\beta$-sheet nucleation and growth are discussed.
\end{abstract}

Keywords: Primary Sequence; Protein Folding; Sequence-Structure Relationship.

\section{Introduction}

It has been almost five decades since Anfinsen and colleagues first confirmed that the folding instructions for ribonuclease A are contained in its primary amino acid sequence [1]. This landmark finding has led to a tremendous amount of research into what has become known as the protein folding problem [2-5], an apt encapsulation for a body of theoretical, biochemical and computational work that aims to unravel the relationship between a protein's primary sequence and its resulting three- 
dimensional structure. In addition to answering fundamental questions about the basic machinery of life - a satisfying enterprise in its own right - a solution to this challenging problem promises to open up tremendous new frontiers in medicine and protein engineering.

Despite substantial theoretical [6-8], experimental [9-11], and computational [12-14] advances, the means by which an unfolded polypeptide chain achieves its final biological conformation continues to evade detailed characterization [13]. Experimentally, the folding process is extremely challenging to study. Important parts of this process, particularly the earliest stages in folding, happen on time scales that are not accessible to almost all biochemical techniques (though there has been considerable progress on this front [15]). In addition, the protein folding data that is available typically pertains to the thermodynamics and kinetics of a whole population of molecules, each following its own particular folding trajectory, with little information available about the specific folding mechanisms themselves [16]. What can be determined from thermodynamic and kinetic results, moreover, suggests that the folding process contains numerous small energy barriers with few - and quite often no - isolatable intermediates [17]. To a large extent, then, the folding process remains a mechanistic "black box" phenomenon, with few specifics established with certainty.

Theoretically, much of the progress in protein folding over the past couple of decades has come from the use of statistical mechanics. Though it has its detractors [18], the Landscape Model of protein folding - which contends that proteins have funneled energy landscapes with many routes down their folding funnels towards their native conformations - has much support within the folding community $[7,8,15,19-21]$. Unfortunately, the theory has yet to foster a breakthrough in protein structure prediction. Of the significant computational hurdles to successful prediction, the most notable one is simply the sheer magnitude of the conformational search. Captured in what is known as Levinthal's paradox, the conformational search that a folding protein must perform to find its native structure, even with substantial simplifications, would take longer than the age of the universe were it done randomly; that most proteins fold on timescales measured in seconds is taken as proof that the folding process cannot be random, but rather must follow some sort of pathway [3]. Yet without the knowledge of folding pathways or even the basic rules of folding, computational methods are often reduced to searching for a needle in an enormous haystack. The most successful approaches to date have made very good use of known protein structures to predict unknown ones, but on the whole, much of their success reflects expertise in copying and not in folding per se [22].

While the protein folding process continues to evade researchers, examples of the end result of this process abound. Indeed, the fact that the protein folding problem remains unsolved has not limited structural biochemists, who have continued to determine protein structures at a staggering pace. As this rich repository of structural information grows, it is natural to wonder whether it can be used to unravel, if not the entire folding process, then at least parts of it, through the identification of statistically significant patterns. It is not surprising, then, that the structural data has been subjected to intense scrutiny for many years, including several attempts to redefine the structural building blocks of proteins [23-25], in the hope of uncovering keys to the relationship between protein sequence and protein structure. Here, we provide an introduction into the use of statistics for finding and analyzing patterns in protein sequences within the context of the protein folding problem. As the sheer magnitude of this topic cannot be covered in detail here, our aim is to focus on the elementary types of statistical research that have been done on protein sequences, and, through highlighting the 
conclusions and interpretations that have been drawn from this research, provide some idea of the power of these basic techniques. Specifically, this survey presents the use of statistics for studying (i) protein primary sequences; (ii) the relationship between protein sequence and secondary structure; and (iii) connections between protein sequence and tertiary structure. Throughout, we discuss the strengths of this approach for studying protein folding, as well as its limitations. As a final example, we close with a re-examination of residue patterning within $\beta$-strands that demonstrates that the type of analysis one performs can directly affect the nature of the results one obtains.

\section{Statistical Analyses of Protein Primary Sequences}

We begin by looking at research that has examined the statistical nature of residue patterning in protein sequences without regards to protein structure. Our focus is on studies of residue patterning, and not on statistical methods for comparing sequences, such as would be found in sequence alignment work. For a recent review of sequence alignment, see [26].

\subsection{The Randomness of the Primary Sequence}

The study of protein sequence data has a long history [27]. One of the earliest analyses of a large ensemble of protein sequences was done by White and Jacobs, who examined the distribution of hydrophobic residues along protein chains [28]. Hydrophobicity has long been considered to be a main driving force behind protein folding [6,29,30], and so one would anticipate that the distribution of hydrophobic residues within protein chains is central to protein folding. By tabulating the frequencies of hydrophobic runs, however, White and Jacobs found that the distribution of hydrophobic residues within protein chains cannot readily be distinguished from random [28]. This study is noteworthy for a number of reasons, not the least of which being the surprising nature of its findings. From a practical point of view, this work demonstrates the use of a very frequently employed simplification in statistical studies: alphabet reduction. Instead of focusing on patterns involving all twenty amino acids, researchers can choose to cluster amino acids together into groupings based on shared physiochemical properties or other features of interest such as homolog substitution frequency [31], local structural environments [32], or tertiary structural environments [33]. In this case, White and Jacobs grouped residues by water affinity into hydrophobic and polar classes. This particular alphabet reduction is used extensively throughout the literature [34], though there is considerable discrepancy with regards to amino acid class assignments, complicating cross-study generalizations. The conclusions from this study are also of particular interest, as they illustrate the scope of statistical implications: White and Jacobs conclude that the random nature of the distribution of hydrophobic residues in proteins chains suggests $(i)$ that functional proteins may have developed from purely random sequences, and (ii) that protein folding may be more permissive with regards to amino acid sequence specificity than is generally thought [28]. This study provides a clear example of how a mix of statistics and deduction can be used to investigate a wide range of protein phenomena, from their distant origins to properties about their folding processes.

The nature of the distribution of hydrophobic residues within proteins has been examined by other researchers, who have drawn different conclusions. Vazquez et al., for example, as well as King and co-workers, concluded after examining such distributions that longer runs of hydrophobic residues 
occur significantly less often than expected [35-37]. The contrast between these conclusions and those of White and Jacobs demonstrate an important characteristic of the use of statistics: different methodologies can lead to different results. For example, Vazquez et al. suggest that their results oppose those of White and Jacobs because of differences between their sets of hydrophobic residues: (VLIFM) in the case of Vazquez et al. [35] as compared to (VLIFMAPWYCG) in the study by White and Jacobs [28]. King and co-workers, on the other hand, have chosen a hydrophobic classification (VLIFMAPWY) [36,37] that is closer to that of White and Jacobs, and so the discrepancy between the major findings of these two studies is unlikely to be the result of different alphabet reduction. Instead, these authors attribute this discrepancy to a subtle difference in calculation methods: in their work, they examine the hydrophobic distributions across their database as a whole, whereas White and Jacobs examine hydrophobic distributions within protein sequences individually [28].

\subsection{Binary Patterns Within Primary Sequences}

Other statistical studies of protein sequences have used a different approach, examining the frequencies of binary patterns along protein chains instead of residue distributions. Vazquez et al., in the same study cited above, looked at the frequency of all possible five-residue binary patterns in their protein dataset, where the binary alphabet was again composed of hydrophobic and polar residues [35]. Those patterns that contained pairs of adjacent hydrophobic residues surrounded by polar ones, or those with hydrophobic residues separated by two polar ones, had frequencies that were statistically higher than expected, while patterns with alternating hydrophobic and polar residues were found to be significantly suppressed. Vazquez et al. cite these findings as further evidence for a non-random distribution of hydrophobic residues within proteins. Moreover, they draw an interesting inference about these types of statistical approaches to the study of protein folding: examinations of patterning within protein sequences without regard to secondary structure may provide insights into folding events prior to secondary structure formation, including the ultra-early events in protein folding. Specifically, they suggest that favoured sequence patterns may have been selected by evolution on the basis of their success at initiating local nucleation events early in the folding process [35]. Hecht and co-workers also found similar results when examining the binary patterning of polar and non-polar residues in proteins $[38,39]$. These authors observed that binary patterns with spacings of polar and non-polar residues matching the characteristic periodicity of particular secondary structures were more likely to be found in those secondary structures, leading them to conclude that the specific binary pattern of a protein sequence can influence the secondary structure which that sequence will adopt. Indeed, in an elegant empirical study that supports their statistical work, they showed that the binary patterning of residues has a greater effect on secondary structure determination than the secondary structural preferences of individual amino acids [40].

As one would expect, it is not just over-represented patterns that are of interest in these studies; those that are under-represented can be equally informative. For example, King and co-workers, in discussing the lower-than-expected number of hydrophobic runs from their analysis, note that it is more likely that these runs are suppressed due to constraints on the folding process rather than on structural constraints on native protein architecture [36]. Specifically, they note that, in concurrence with prior simulation results, hydrophobic runs may be selected against by evolution because they may 
promote off-pathway aggregation during folding [36,37]. Hecht and co-workers draw similar conclusions from the lower-than-expected frequencies of alternating polar-nonpolar patterns found in their work and others [39]. These alternating patterns might be thought to be favoured $a$ priori, given that their periodicity matches that found in amphipathic $\beta$-strands. However, as patterns of alternating polar and nonpolar residues are known to promote amyloid-like structure in designed proteins, Hecht and co-workers suggest that alternating patterns are also subjected to negative selection by evolution to reduce amyloidogenesis during the folding process $[38,40]$. In both of these classes of studies, the statistically low results have been taking as an indication of negative design, a term referring to the need for protein sequences to not only code for the correct native structure, but also to code against competing structures. Schwartz and King, extrapolating from their statistical analyses, put it as follows: "amino acid sequence must be treated not merely as a "structural code" encoding a protein's native state but also as a "folding code" providing additional information on how to get to and remain in the native state while avoiding pitfalls such as aggregation" [37].

\subsection{Analyzing the Primary Sequence Using Information Theory}

We conclude this section with a brief description of one further whole-sequence method that uses a different approach to sequence analysis: information theory. From an information point of view, protein folding can be seen as the transfer of information from one-dimensional chains of amino acids to three-dimensional folded proteins. In this context, Strait and Dewey [41], as well as Weiss et al. [42], have estimated the amount of information contained in protein sequences using Shannon information entropy [43]. Entropy, within information theory, is a widely-used measure expressed as the number of bits needed to store a set of data, and as such, it is inversely related to compressibility and redundancy. Random datasets, with minimal redundancy, have maximal entropy, and carry the maximum amount of information per unit of data. Weiss et al. note that it is very difficult to determine a reliable estimate of the sequence entropy because of the finite nature of the data been studied [42]. To circumvent this inherent limitation, they employ several different alphabet reductions to improve their statistics, from which they conclude that protein sequences are very nearly random, containing approximately $99 \%$ of the complexity of random sequences [42]. Strait and Dewey, on the other hand, drew different conclusions. Faced with the same problem of finite data, they used a variety of different techniques to corroborate their estimation that amino acids carry between $2.4-2.6$ bits of information per amino acid, substantially less than the $4.2-4.3$ bits of information carried by amino acids in random sequences [41]. These opposing results reflect another pitfall of statistical approaches: they may be based on underlying assumptions or have fundamental requirements that cannot easily be satisfied. In this case, the application of Shannon entropy analysis is compromised by the limited amount of data [42]. Moreover, this technique can produce varying results depending upon the extent of its application, and corroborating evidence may be needed to increase the reliability of the results. While information theory offers novel techniques for studying protein sequences, thus far it has been unable to unambiguously determine the degree of randomness in protein sequences. 


\section{Primary Sequence and Secondary Structure}

\subsection{Amino Acid Conformational Propensities}

The statistical study of secondary structural sequence data began with the seminal study by Chou and Fasman that analyzed the residues within helices, $\beta$-structures and coil regions of 15 of the earliest available protein structures [44]. Despite the limited size of the dataset in this study, it was already evident that amino acids are not distributed randomly amongst the various types of secondary structure. These authors introduced the statistical term conformational parameter to describe the propensity for a residue to be found in a particular secondary structural environment. Conformational propensity values, which have come to be widely used throughout the literature, are normalized frequency values: the propensity for residue $r$ in secondary structure $S S, P_{r, S S}$, is given by:

$$
P_{r, S S}=\left[S S_{r} / S S_{\text {all }}\right] /\left[\text { Total }_{r} / \text { Total }_{\text {all }}\right]
$$

where $S S_{r}$ and $S S_{\text {all }}$ are the number of $r$ residues, and of all residues, in secondary structure $S S$, and

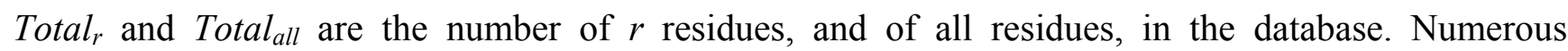
subsequent studies of secondary structure propensity values for $\alpha$-helices [45-52], $\beta$-sheets [53-55], turns [56-59] and other secondary structural elements [60-63] have appeared in the literature, with general agreement. Ala, Glu, Met and Leu, for example, have high helix propensity values, while the short polar residues Ser, Thr and Asn, together with Gly and Pro, have low ones [51]. In contrast, these residues have approximately reversed propensities for turns [44].

Following the advent of additional structural data, more specialized statistical analyses became possible. Two groundbreaking papers published in the late 1980's focused on residue positional preferences within helices. In one, Richardson and Richardson, using a set of $215 \alpha$-helices from 45 globular protein structures, took the notion of conformational propensities one step further and calculated position-specific residue propensities for a number of positions at both the $\mathrm{N}$ - and $\mathrm{C}$ terminal (NT and CT) ends of helices [45]. As has been confirmed by numerous subsequent studies [46-48,50,64], short polar residues, along with Gly, are strongly favoured just prior to helices, while Pro, Glu and Asp are strongly favoured in the first few NT helical positions. Gly, on the other hand, is overwhelmingly favoured just after the last helical position, a location almost entirely devoid of Pro residues. At the same time, Presta and Rose, from a consideration of the incomplete hydrogen bonding patterns at the two ends of helices, proposed the helix hypothesis [65]. This hypothesis postulates that a necessary condition for helix formation is the presence of polar residues in the flanking regions around helices that can help to complete the missing hydrogen bonds at helical termini, thereby stabilizing nascent helices. Collectively, this research provides a prime example of how statistical results can foster empirical work: the discovery of helix capping - the sequential and structural motifs at the two helical termini that promote hydrogen bonding and hydrophobic interactions between the terminal helical residues and those immediately flanking the helical ends - has precipitated a large body of experimental research into the role of capping in helix stability [66-71]. Helix capping has also been included in both theoretical aspects of protein folding [72] and prediction methods [73]. In a broader sense, the idea of position-specific propensity values has been used to uncover residue preferences in other secondary structural environments, including at the termini of $\beta$-strands [60], in $\beta$ bulges [61], and within turns [56-59], among other environments [62]. 
As with studies of the primary sequence, a combination of statistical analyses of secondary structure and careful deduction can lead to inferences about the protein folding process itself. A clear example of this is found in two ingenious statistical studies of helices and turns by Chakrabarti and colleagues [50,59]. These authors examined the positional propensities of residues in the smallest helices (containing either three or four residues), and compared them with the positional propensities of residues in longer $\alpha$-helices, and also with positional propensities of residues in $\alpha$ - and $\beta$-turns. The choice to examine only the smallest helices is noteworthy: as helical growth is thought to consist of an initial nucleation step followed by an elongation step, the authors hypothesized that the smallest helices have undergone nucleation only, and not subsequent growth. These short helices, therefore, may provide a means of identifying helix nucleation sites for subsequent analysis. Chakrabarti and colleagues [50] report a very high correlation between the residue propensities in the shortest helices and those found at the NT, but not the CT, end of longer $\alpha$-helices, from which they draw two interesting implications for protein folding: (i) helix nucleation might occur predominantly at the $\mathrm{N}$ terminus of helices; and consequently (ii) helical growth might be largely unidirectional, from NT $\rightarrow$ CT. Moreover, high correlations between the residues involved in the shortest helices and those found in both $\alpha$ - and $\beta$-turns led the authors to propose that $\alpha$-helices might originate from $\beta$-turns, with either $\alpha$-turns or short $3_{10}$-helices acting as intermediaries [50,59]. It is noteworthy that, though the NT of helices has been suggested by others to be involved in helix nucleation [74], these statistical studies have provided the only evidence to date in support of this proposition.

\subsection{Residue Coupling}

Residue coupling analysis investigates how residues pair together in various sequential relationships within secondary structures. A central hypothesis of this type of analysis is that the energetic effects of various types of pairing interactions will be reflected in a Boltzmann-like distribution of these pairings within a large, random sample of structures [75]. Thus, statistically elevated (reduced) pairing frequencies are interpreted to reflect favourable (unfavourable) underlying interactions [52]. In the case of helices, pairing preferences for various spacings have been tabulated, either along helices as a whole [27,51,76-80], or at helical termini [27,52]. The dominant pattern exhibited within helices reflects amphipathicity: the tendency for polar and non-polar amino acids to be segregated on opposite sides of a helix [76]. High coupling rates for pairs of hydrophobic residues, as well as pairs of hydrophilic residues, have been reported at $(i, i+3)$ and $(i, i+4)$ spacings within helices, precisely the spacings that bring residues next to one another spatially on a single helical face. Similarly, low coupling rates are reported at the $(i, i+2)$ opposing-helical-face spacing. Pairings at the $(i, i+3)$ and $(i, i+4)$ spacings have received the lion's share of attention, as these spacings capture the effects of side-chain-side-chain (SC-SC) interactions within helices [77-80]. Some studies use statistical coupling to test existing hypotheses about particular SC-SC interactions [77]. Most studies, however, perform what might be termed a statistical "fishing" exercise. Here, coupling analysis is performed on all possible pairings, and statistically significant ones are identified. Andrew et al., for example, identified higher-than-expected pairing frequencies between polar and non-polar amino acids at the $(i, i+4)$ same-helical face spacing, in contradiction to the dictates of amphipathicity [80]. The authors went on to verify this curious observation experimentally, thereby providing the first proof of 
polar-nonpolar stabilizing interactions in helices [80]. In some studies, a lack of correspondence between statistics and empirical observations was the dominant observation. Fernández-Recio and Sancho found poor correlation between pairing frequencies and measured SC-SC interaction energies in short helical peptides [79]. Their conclusions about this unexpected finding are noteworthy, and again demonstrate the type of inferences about protein folding that can be drawn from statistical work. Specifically, they conclude that the lack of correlation between SC-SC interaction energies and occurrence frequencies suggests that SC-SC interactions are poorly exploited by evolution for increasing helical stability [79]. They suggest that this may be because of: (i) an elevated role for tertiary interactions in helix stability, a proposition that lends support to folding models where secondary and tertiary folding are mutually stabilizing; and/or (ii) a requirement that proteins remain marginally stable for functional reasons, something that can be achieved if stabilization strategies are under-employed within protein structures. Indeed, despite clear amphipathic helical patterning, others have also questioned the importance of SC-SC interactions for helix stability [78].

Secondary structural coupling analysis has been used to study residue patterning in virtually all secondary structural environments. The residues in various turn positions, for example, have been examined for pairing preferences [59], as have the residues in helical capping positions [27,52] and loop regions $[27,63]$. Ordering within pairings has been explored, and pairing preferences have been found to be non-symmetrical [52]. But amongst the various secondary structural environments, it is $\beta$ architecture that has received the greatest attention from coupling analysis, most likely because so little is known about its folding process. Numerous studies have looked at coupling between both inter- and intra-strand pairs of residues within $\beta$-sheets in an effort to identify possible sequential motifs that might explain sheet origins [27,53-55,81-84]. Early studies showed that inter-strand $\beta$-sheet pairings favoured electrostatic and hydrophobic compatibility [81], though in a non-specific manner. Lifson later showed that inter-strand pairing preferences were actually specific: Ser/Thr, Ile/Val, Ile/Leu and (Lys,Arg)/(Asp,Gln) were all found to have higher-than-expected pairing frequencies between strands [82]. Pairs of Cys residues have also been reported to occur more often than expected in neighbouring cross-strand positions [54,84]. Moreover, the complexities of $\beta$-architecture have allowed for more specific coupling analyses: inter-strand pairing preferences have been calculated separately for parallel and anti-parallel strands [27,54,83,84], for edge and interior strands [27], and for hydrogen bonded and non-bonded positions within strands [27,54,83,84]. Following from these statistical studies, the conformations adopted by interacting, cross-strand side-chains have been investigated $[54,83,84]$. Characteristic conformations for specific residue pairings are commonly seen, each with intimate SC-SC contacts between the residues that often involve hydrogen bonding and nesting along the length of the side-chain that promotes van der Waals interactions. Interestingly, a number of coupling studies of intra-strand pairings did not find any statistically significant variations from random in $(i, i+2)$ residue pairings [81,83], a somewhat unexpected finding given that this spacing will place two residues spatially next to one other on the same side of a $\beta$-sheet. The distance between these pairings is generally thought to be too great to allow for such $(i, i+2) \mathrm{SC}-\mathrm{SC}$ interactions $[81,83]$.

As with other types of statistical analyses, the examination of residue coupling within secondary structures has lead to general inferences about the process of protein folding. In a frequently cited study, for example, Curmi et al. found statistical evidence suggesting that electrostatic compatibility 
was a key component in the pairing preferences of residues on adjacent $\beta$-strands [54]. However, they did not observe any particular electrostatic SC-SC interactions between strands within protein structures that would account for the elevated electrostatic pairing frequencies. The authors solve this potential disparity in their findings by moving beyond protein structure: they propose that the elevated electrostatic pairing frequencies do not reflect contributions to structural stability of $\beta$-sheets, but rather contributions to the process of $\beta$-structure formation itself. Specifically, they posit that electrostatic interactions may be critical for establishing the correct strand-strand register, or alignment, early in sheet development [54]. Such initial aligning SC-SC interactions, they contend, may not necessarily carry forward to final protein tertiary structure. In another study, MandelGutfreund et al. draw contrasting conclusions about sheet formation through the use of a different statistical approach: conservation and covariance [85]. In this case, the authors do not analyze pairing preferences in a non-redundant dataset, but instead look at how individual amino acids in pairs of neighbouring residues on adjacent strands have mutated within families of evolutionarily related proteins. They report that, while buried pairs are more conserved than those on protein surfaces, the degree of covariance and conservation observed between neighbouring residues on adjacent strands, regardless of whether they are buried or not, is not statistically different than that observed between other, non-neighbouring pairings [85]. From this counter-intuitive observation, the authors conclude that the evolutionary pressure for residue complementarity between pairs on adjacent strands is weak [85]. Sheet formation, they suggest, is not as dependent on specific SC-SC interactions after all.

\section{Primary Sequence and Tertiary Structure}

It is widely recognized that the relationship between a protein's primary sequence and its tertiary structure is based on a combination of both secondary and tertiary interactions [86], and as such, no simple connection between the two is expected. Overarching rules - if they exist - are extraordinarily elusive. A number of inferences about protein folding from studies of the primary sequence alone and in relation to secondary structure have been presented in previous sections. Here, we conclude this survey of the statistical study of protein sequences by examining how sequence analysis has been used within three important fields of tertiary structure research: separating structured from unstructured proteins, identifying domain boundaries within structured proteins, and predicting the structural classes of protein domains.

\subsection{Natively Disordered Proteins}

There is now abundant evidence that a substantial portion of proteins, particularly in eukaryotes, lack definitive structure under physiological conditions, yet these disordered, or natively unfolded, proteins are increasingly found to have important functional roles within cells [87-89]. The discovery of functional proteins that lack stable, tertiary structure also adds a level of complexity to the sequence-structure relationship, as it divides the population of sequences into two: those that yield natively ordered, and those that yield natively disordered, proteins. This development serves as a reminder that the protein structures solved to date - and therefore the statistics that have been derived from them - do not necessarily reflect the characteristics of all proteins, but rather are biased towards those proteins that are amenable to structure determination, namely those that can be cloned, 
expressed, purified and solved by X-ray crystallization or NMR. Moreover, this novel class of proteins can also serve to remind about the importance of flexibility for protein architecture and interactions, something that can easily be lost when considering the more rigid concept of protein 'structure.' Because of the clear structural and functional differences between these two classes of proteins, it is natural to ask whether there are any sequential differences between them. As in other areas of protein folding studies, statistical analysis has been employed to address this question. Uversky et al., for example, have used comparative statistics to search for differences in the sequences of these two populations [90]. While characteristics such as polypeptide length, net charge and $\mathrm{pI}$ were not found to differentiate between natively folded and unfolded proteins, these authors demonstrated that a combination of mean net charge and mean hydrophobicity can be used to distinguish between them. The authors further suggest that natively unfolded proteins can be induced to adopt folded conformations through interactions with other proteins or ligands that produce an increase in the mean hydrophobicity and a decrease in the mean net charge in the resulting complex [90]. This work therefore supports a model of protein folding that is based on the creation of a stabilizing hydrophobic core built from residues located, not in isolated segments of a chain, but throughout the entire chain length. In addition, others have shown that disordered proteins have lower sequence complexities, as measured using information theory [91], and have differences in their amino acid composition [91,92], than are found in natively ordered proteins. Thus, while this is a relatively new field of study within the larger context of protein folding, the importance of statistical analysis to this field is already evident.

\subsection{Domain Boundary Prediction}

A related area of study within protein folding is domain boundary prediction. Domains are autonomously folding units within proteins [93,94] that are separated from one another by linker regions. These linker regions are often disordered, and are thus structurally similar to natively unfolded proteins. While most techniques for identifying domain boundaries make use of evolutionary information [94,95] or machine learning techniques [96-99], there have been several statistical attempts in recent years to identify domain boundaries by direct examination of a protein's amino acid sequence [100-102]. Most have combined structural insights with relatively simple statistical measures to achieve reasonable success at boundary prediction. For example, Wheelan et al., noting the narrow range in the number of residues observed in domains of solved proteins, achieved surprisingly successful boundary prediction by making what amounts to educated guesses, using the statistical distribution of domain lengths to guide their predictions [100]. Galzitskaya and Melnik, on the other hand, used a statistical approach to domain boundary prediction that was grounded on an interesting premise: they hypothesized that, for protein folding to occur, the amount of entropy lost along a protein chain during folding (approximated for each residue by the number of dihedral angles in its backbone and side-chain) must be compensated for by localized enthalpy gains [102]. Given this, the authors conclude that those parts of a chain with high potential for entropy loss will be the primary loci of folded domains, as the domain architecture will provide the close packing in these regions required to offset their high entropy losses. Using this, they built a predictive scheme that statistically scored the entropy within a sliding window of residues, and predicted domain boundaries at entropic 
minimums [102]. Finally, other methods have based their predictions on statistical differences in amino acid composition between linker regions and folding domains [101], with some success.

\subsection{Domain Structural Class Prediction}

Domain structure is another area of protein research that has received considerable attention. At the atomic level, no two domain structures are the same, but looked at from a topologically higher level, structural patterns begin to emerge. An early study by Levitt and Chothia [103] found that domains could be separated into four structural classes: all- $\alpha$, all- $\beta, \alpha+\beta, \alpha / \beta$. This basic classification is still widely used today. For a protein with unknown structure, knowledge of its structural class is of importance for numerous reasons [104], not the least of which is that this information can greatly improve the accuracy of structure prediction. Consequently, structure class prediction has been an active area of research for many years, with statistical approaches used extensively [104-109]. An early study by Nakashima et al. analyzing the amino acid composition of proteins in different structural classes showed that proteins tended to cluster into their own regions of 20-dimensional space, showing directly that a protein's amino acid composition (AAC) is correlated to its structural classification [105]. This significant finding led to the development of a number of prediction techniques based on the statistical differences in AAC across structural classes [104,106,107]. Chou and Zhang [104] improved the predictive power of AAC methods by introducing a new distance metric into the analysis, the Mahalanobis distance [110], which included coupling, or statistical covariance, between amino acids. Others expanded on the basic approach by replacing AACs with distributions of physiochemical properties of the amino acids [106]. Hybrid approaches have also been reported [107]. In general, all of these methods exploit the statistical relationship between the amino acid composition of a protein and the structural fold of that protein, though the specific nature of this relationship remains unknown. Other groups have used simpler statistical methods to achieve considerable predictive success, relying on differences in the occurrence frequencies of di-, tri- and quad-peptides within the different structural classes for making predictions [108,109].

\section{A Novel Example: Residue Patterning in $\beta$-Strands}

We conclude this introduction to the statistical study of residue patterning within protein sequences by reporting novel findings from our lab regarding residue patterning within $\beta$-strands. To better illustrate how the use of statistics can aid in investigating problems associated with protein folding, we first briefly describe the motivations of this present work.

It has long been postulated that proteins undergo a transition from the unfolded to the folded state as a result of hydrophobic collapse $[6,29,30]$. Virtually all globular proteins fold so that the majority of their hydrophobic residues become buried in their core, thus reducing their exposure to water [111]. Although the specific mechanism(s) leading to the formation of $\beta$-structures are not known [112], this general principle of hydrophobic sequestration has also been proposed to account for the formation of $\beta$-sheets [6,113]: hydrophobic residues on separate strands make initial contacts to form hydrophobic clusters, and sheet formation ensues around these clusters. This model requires specificity, both in terms of a non-random distribution of key hydrophobic residues in and around $\beta$-strands, and in terms of the residues surrounding these key hydrophobic residues. Without such specificity, there will not be 
a way for native, on-pathway hydrophobic contacts to distinguish themselves from non-native, offpathway ones. Thus, under this folding model the local patterning of hydrophobic residues in and around $\beta$-strands is critical for achieving correct native folds.

Given this model for $\beta$-sheet nucleation that involves both hydrophobicity and residue specificity, we hypothesize that the distribution of hydrophobic residues within $\beta$-strands is not random, but rather must contain local aggregations that provide the specificity necessary for correct strand-strand recognition during $\beta$-sheet nucleation. We are therefore chiefly interested to see if there is an increased frequency of $(i, i+2)$ same-sheet-surface hydrophobic pairings on $\beta$-strands. As outline in previous sections, numerous studies of hydrophobic patterning within $\beta$-sheets and within protein sequences generally have appeared in the literature [28,35-40,54,55,81-84]. Some find that protein sequences are essentially random [28], while others do not [35-40]. Specifically with regards to $\beta$-strands, two studies have reported that the frequency of $(i, i+2)$ pairing cannot be distinguished from random $[81,83]$, contrary to our hypothesis. To investigate this further, we have re-examined the coupling of hydrophobic residues in $\beta$-strands anew, this time extending the analysis of previous works by looking at position-specific residue coupling.

\subsection{Methods}

For our investigation, we compiled a non-redundant dataset of protein structures using the PDBREPRDB database [114], which consists of representative protein chains from the RCSB Protein Data Bank, release \#2007_01_27. Only protein chains solved by X-ray crystallography with resolutions less or equal to $2.0 \AA$, and R-factors less than or equal to 0.25 were considered. In addition, to be included, protein chains had to be free of chain breaks, have coordinates for all non-hydrogen atoms, and be at least 40 residues in length. Membrane proteins, as well as those polypeptide chains that were part of larger structural complexes, were discarded. To ensure non-redundancy in our dataset, only one representative was chosen from amongst those protein chains that had sequence identities greater than $30 \%$, or structural alignments less than $10 \AA$. In all, our dataset consisted of 1338 protein chains. $\beta$-strands were identified in our non-redundant dataset using the program DSSP [115]. A total of 11,682 strands of length 3 or more were identified and used in our study, of which 3565 were parallel and 7023 were anti-parallel.

With this dataset, we calculated coupling statistics for pairs of hydrophobic residues (VLIFM), and pairs of hydrophilic residues (KREDSTNQ), at various spacings in $\beta$-strands. Expected values and standard deviations were calculated using a shuffling procedure as follows. For each query pattern, the actual frequency was first tabulated within our dataset. Following this, the amino acids within all strands of sufficient length (ie. all strands at least as long as the query pattern) were redistributed randomly 1,000 times, and a new count of the query pattern frequency was made after each randomization. The resulting 1,000 counts were used to determine an expected value and a standard deviation for the query pattern, providing a means of evaluating the actual frequency count. Statistics were calculated separately for (i) all strands, (ii) parallel strands, and (iii) anti-parallel strands. 


\subsection{Results and Discussion}

Using $z$-scores to compare measured and expected frequencies, we find that both hydrophobic and hydrophilic pairings occur with non-random frequencies in $\beta$-strands (Table 1, Figure 1). Hydrophilic pairs exhibit clear amphipathic patterning at the $(i, i+1)$ through $(i, i+4)$ spacings, while at further spacings these pairings occur more often than expected, though the statistical significance tapers towards randomness at larger spacings. The same trends hold for pairs of charged residues (KRED) and neutral hydrophilic residues (STNQ), calculated separately (data not shown). Hydrophobic pairs, in contrast, are found with low frequencies at virtually all spacings, implying that the distribution of hydrophobic residues within $\beta$-strands does not reflect amphipathicity. Pairs of hydrophobics are particularly suppressed at $(i, i+1),(i, i+3)$ and $(i, i+4)$ spacings, with calculated $z$-scores of $-20.3,-20.0$ and -20.1 respectively. The values for the $(i, i+1)$ and $(i, i+3)$ spacings suggest that hydrophobics are strongly discouraged from being on opposite sides of $\beta$-sheets, consistent with amphipathicity, but the marginally elevated $(i, i+2)$ value $(z$-score 2.2$)$ and the highly suppressed $(i, i+4)$ do not suggest that hydrophobic residues are encouraged to be near one another on the same $\beta$-surface.

Table 1. Coupling analysis between pairs of hydrophobic (VLIFM), and pairs of hydrophilic (KREDSNTQ), residues at various spacings within $\beta$-strands.

\begin{tabular}{c|ccc|ccc}
\hline & \multicolumn{3}{|c|}{ Hydrophobic } & \multicolumn{3}{c}{ Hydrophilic } \\
Spacing & Frequency & Expected & Z-score & Frequency & Expected & Z-score \\
\hline & & & & & & \\
$i+1$ & 9361 & 10592.9 & -20.3 & 4579 & 5751.3 & -22.2 \\
$i+2$ & 8291 & 8161.8 & 2.2 & 5467 & 4716.4 & 15.1 \\
$i+3$ & 4803 & 5787.4 & -20.0 & 3475 & 3614.1 & -3.2 \\
$i+4$ & 2871 & 3759.1 & -20.1 & 2950 & 2714.0 & 5.9 \\
$i+5$ & 1960 & 2258.3 & -8.6 & 2197 & 1983.0 & 6.5 \\
$i+6$ & 1133 & 1322.7 & -6.6 & 1526 & 1339.4 & 6.8 \\
$i+7$ & 738 & 758.4 & -1.0 & 988 & 889.8 & 4.3 \\
$i+8$ & 386 & 428.4 & -2.6 & 615 & 571.8 & 2.5 \\
\hline
\end{tabular}

Figure 1. Hydrophobic and hydrophilic coupling within $\beta$-strands.

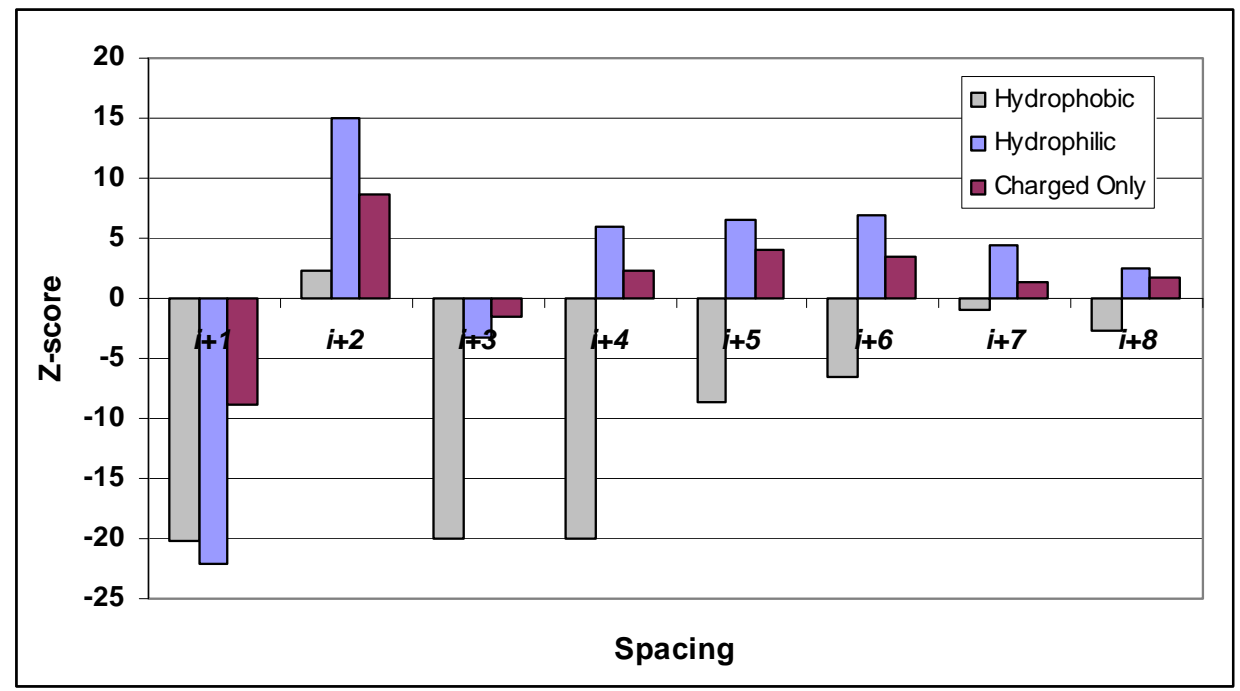


These results imply that residue distributions in $\beta$-architecture are non-random. However, at the critical $(i, i+2)$ spacing that is the focus of this investigation, there is less certainty. Hydrophilic residue pairs occur at this spacing with frequencies much higher than would be expected if the hydrophilic distribution was random; however, the frequency of hydrophobic pairings is much closer to what would be expected for a random hydrophobic distribution. This concurs with an early study by Heijne and Blomberg [81], as well as a more recent one by Hutchinson et al. [83], who found no evidence of $(i, i+2)$ hydrophobic coupling. To investigate this further, we extended our coupling analysis by examining position-specific coupling at various positions relative to the NT and CT ends of strands. Though other types of secondary structure have been so studied, to our knowledge position-specific coupling analysis has not been applied to $\beta$-architecture. Table 2 and Figure 2 contain the positionspecific $(i, i+2)$ pairing frequencies for hydrophobic residues at positions ranging from $(N T, N T+2)$ through $(N T+6, N T+8)$, as well as for positions ranging from (CT-8,CT-6) through $(C T-2, C T)$. From this figure, it is evident that the frequency of hydrophobic $(i, i+2)$ pairings is not uniform across all positions. Instead, there are high levels of hydrophobic coupling at the two termini, particularly at the $(N T+1, N T+3)$ position, and at the $(C T-4, C T-2)$ and $(C T-2, C T)$ positions. In contrast, at central positions the coupling frequency cannot be distinguished from what would be expected from a random hydrophobic distribution. Moreover, the frequencies of hydrophobic $(i, i+2)$ pairings differ markedly between parallel and anti-parallel strands. On parallel strands, $(i, i+2)$ pairings of hydrophobic residues occur with high frequency at the $(N T+1, N T+3)$ position, and show only marginally increased frequencies at the CT. On the other hand, hydrophobic $(i, i+2)$ pairs are found with high frequencies on anti-parallel strands in tandem positions at both the NT and CT: positions $(N T+1, N T+3)$, $(N T+3, N T+5),(C T-4, C T-2)$ and $(C T-2, C T)$ all have high hydrophobic pairing frequencies. In short, parallel strands have increased hydrophobic pairing only at their NT, while anti-parallel pairs have increased hydrophobic pairing at both of their termini. Interestingly, if one considers the orientations of strands, the increased hydrophobic pairings at both ends of anti-parallel strands may simply reflect hydrophobic patches at one end of anti-parallel strand pairs, a conjecture that is supported by the curious finding of increased hydrophobic pairings at tandem positions of both anti-parallel termini. Under this interpretation, pairs of both parallel and anti-parallel strands can be seen to have increased hydrophobic pairing at one of their ends.

We therefore find evidence of increased hydrophobic $(i, i+2)$ pairings in $\beta$-strands. These results differ from those of early studies [81,83], but this difference can be attributed to the uneven distribution of such pairings on strands: there are specific locations at strand termini that show strong $(i, i+2)$ hydrophobic coupling, while frequencies at other locations suggest that no such coupling exists.

Of note about this and other statistical techniques that use a large, non-redundant dataset of protein structures to investigate the sequence/structure relationship is that the findings should be independent of the structural peculiarities of any of the specific proteins in the dataset. Thus, in this case, our findings reflect the characteristics of an idealized $\beta$-strand, created from an average over all the strands in our dataset. The effects of interesting structural phenomena, such as $\beta$-bulges which would reverse the strand sense, will not detract from the major coupling patterns found on $\beta$-strands, providing the occurrence of unusual structural phenomena such as $\beta$-bulges is randomly distributed along strands. 
Table 2. Position-specific coupling analysis between pairs of hydrophobic (VLIFM) residues across all strands, parallel strands, and antiparallel strands.

\begin{tabular}{c|ccc|ccc|ccc}
\hline & \multicolumn{3}{|c|}{ All Strands } & \multicolumn{3}{c}{ Parallel Strands } & \multicolumn{3}{c}{ Antiparallel Strands } \\
Pairing & Freq. & Expect & Z-score & Freq & Expect & Z-score & . & Expect & Z-score \\
\hline & & & & & & & & & \\
NT,NT+2 & 1976 & 1835.1 & 4.6 & 802 & 748.1 & 3.0 & 952 & 944.9 & 0.3 \\
NT+1,NT+2 & 2450 & 1937.3 & 14.2 & 1004 & 853.4 & 7.5 & 1181 & 948.7 & 9.8 \\
NT+2,NT+4 & 1463 & 1408.7 & 1.8 & 522 & 519.8 & 0.1 & 718 & 743.6 & -1.1 \\
NT+3,NT+5 & 1086 & 916.2 & 6.6 & 244 & 243.2 & 0.1 & 685 & 551.9 & 6.8 \\
NT+4,NT+6 & 498 & 550.7 & -2.7 & 81 & 96.9 & -2.1 & 348 & 370.4 & -1.3 \\
NT+5,NT+7 & 345 & 324.8 & 1.3 & 31 & 31.2 & 0.0 & 264 & 239.1 & 1.8 \\
NT+6,NT+8 & 191 & 180.8 & 0.9 & 11 & 13.0 & -0.6 & 137 & 134.5 & 0.2 \\
& & & & & & & & & \\
CT-8,CT-6 & 168 & 189.7 & -1.9 & 14 & 13.1 & 0.3 & 127 & 142.1 & -1.4 \\
CT-7,CT-5 & 339 & 338.0 & 0.1 & 32 & 34.2 & -0.5 & 245 & 248.4 & -0.3 \\
CT-6,CT-4 & 571 & 571.1 & 0.0 & 102 & 105.8 & -0.5 & 379 & 379.0 & 0.0 \\
CT-5,CT-3 & 995 & 947.5 & 1.9 & 268 & 259.7 & 0.7 & 593 & 565.3 & 1.4 \\
CT-4,CT-2 & 1722 & 1439.1 & 9.1 & 568 & 539.4 & 1.7 & 910 & 753.7 & 6.7 \\
CT-3,CT-1 & 2204 & 1958.5 & 7.0 & 925 & 859.1 & 3.2 & 1029 & 961.3 & 2.7 \\
CT-2,CT & 2032 & 1719.0 & 10.5 & 789 & 735.6 & 3.0 & 1021 & 857.9 & 7.2 \\
\hline
\end{tabular}

Figure 2. Position-specific hydrophobic coupling within $\beta$-strands.

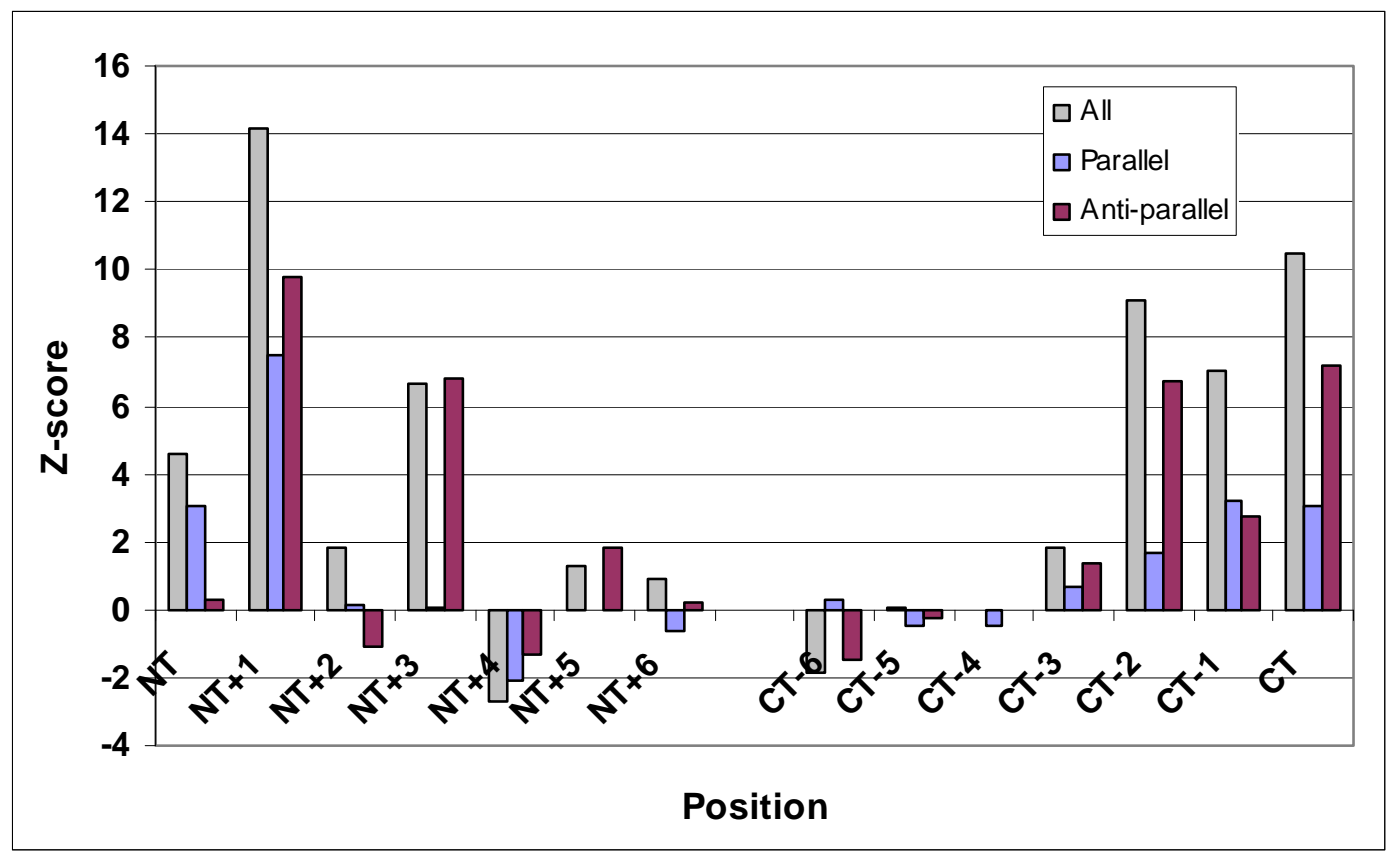

As with any statistical result, there is always the question of interpretation. In this case, we interpret our results to support a model of sheet nucleation that involves hydrophobic interactions between disparate parts of the protein chain. As mentioned, such a model requires specificity: non-native sheet nucleation can impair protein folding and lead to aggregation problems such as is seen in amyloidogenesis [116]. Indeed, others have speculated that the low frequency of $(i, i+2)$ hydrophobic pairings across protein sequences reflects the principle of negative design in evolution $[38,40]$ which seeks to minimize inadvertent sheet formation. In this light, it is not surprising to find that $\beta$-sheets do 
not have an elevated frequency of $(i, i+2)$ hydrophobic pairs. However, as native sheets also require nucleation, a localized increase in $(i, i+2)$ hydrophobic pairings can still be expected. Our positionspecific analysis finds precisely this: increased hydrophobic pairings at strand termini. One possible explanation for our findings, therefore, is that the sites of sheet nucleation are located predominantly at the edges of sheets. A corollary to this is that, following nucleation between two disparate parts of the chain, the majority of $\beta$ growth between these chains is unidirectional. For parallel strands, this direction can be specified as $\mathrm{NT} \rightarrow \mathrm{CT}$. Moreover, it is curious that the increased $(i, i+2)$ hydrophobic coupling occurs at the $(N T+1, N T+3)$ position, and not the $(N T, N T+2)$ position. It may be that the NT position directly before a nucleation seed is itself involved in the seed (thereby conferring additional specificity) in such a way as to block subsequent growth towards the chain NT.

While this interpretation is consistent with our hypothesis, it must be remembered that statistics alone cannot provide physical explanations. Lifson noted as such many years ago: "[t]he physical reasons for the particularly favourable or unfavourable pairs are impossible to determine by statistical analysis" [82]. Another interpretation of our results, for example, can be traced back to an early study of sheet architecture by Cohen et al. These authors, when studying $\beta$-sheet sandwich architecture, noted that the major hydrophobic interactions between sheets occur at the sheet corners [117]. Although their study is limited to a single class of $\beta$-architecture, it may be that this is a wider phenomenon, and that the increased hydrophobic pairing that we find at strand termini is a reflection of such a phenomenon. We are presently extending our work beyond single strands to investigate this possibility, as well as the nature of the hydrophobic distribution across sheets in general.

\section{Concluding Remarks}

We have attempted to give a general introduction into the use of statistics for studying residue patterning within protein sequences. As this is an extended topic, we have aimed at breadth rather than depth, though even covering the full breadth of statistical pattern mining in protein chains is a very large undertaking. Consequently, we have chosen those areas of research that we feel best exemplify the power of statistical analyses for drawing inferences about events that are difficult or even impossible to study empirically, such as early events in protein folding or details about the evolutionary origins of modern-day proteins.

Statistical analyses provide a powerful way of summarizing properties of protein sequences that can easily go unnoticed, particularly when attention is focused on a single or a small number of protein structures. As such, a broad survey of protein sequence and structure may uncover connections that can then be investigated experimentally. Part of their utility comes from their ease of use: it is almost always far easier to analyze a set of protein sequences then devise and implement a series of experiments to study a particular phenomenon.

Despite its utility, there are important limits to what can be accomplished with statistics. The interpretation of results is an important part of research, but it must be remembered that such efforts are solely inferences. Cause and effect are often difficult to untangle. We conclude by revisiting the words of Lifson and Sander, which aptly describe the limits of statistical analysis: 
"... the limitations in all statistical approaches to protein structure must be kept in mind: proteins are biologically evolved molecules with a particular structure tailored to a particular function. Only average properties of protein structures can be understood by statistical methods, but not the highly individual character of each protein" [53].

\section{References and Notes}

1. Anfinsen, C.B.; Haber, E.; Sela, M.; White, F.H.Jr. The kinetics of formation of native ribonuclease during oxidation of the reduced polypeptide chain. Proc. Natl. Acad. Sci. USA 1961, 47, 1309-1314.

2. Rossmann, M.G.; Argos, P. Protein folding. Ann. Rev. Biochem. 1981, 50, 497-532.

3. Levinthal, C. Are there pathways for protein folding? J. Chem. Phys. 1968, 65, 44-45.

4. Fetrow, J.S.; Giamonna, A.; Kolinski, A.; Sholnick, J. The protein folding problem: a biophysical enigma. Curr. Pharm. Biotechnol. 2002, 3, 329-347.

5. Dill, K.A.; Ozkan, S.B.; Shell, M.S.; Weikl, T.R. The protein folding problem. Annu. Rev. Biophys. 2008, 37, 289-316.

6. Dill, K.A.; Fiebig, K.M.; Chan, H.S. Cooperativity in protein-folding kinetics. Proc. Natl. Acad. Sci. USA 1993, 90, 1942-1946.

7. Onuchic, J.N.; Wolynes, P.G. Theory of protein folding. Curr. Opin. Struct. Biol. 2004, 14, 70-75.

8. Wolynes, P.G. Energy landscapes and solved protein-folding problems. Phil. Trans. R. Soc. A 2005, 363, 453-467.

9. Marqusee, S.; Robbins, V.H.; Baldwin, R.L. Unusually stable helix formation in short alaninebased peptides. Proc. Natl. Acad. Sci. USA 1989, 86, 5286-5290.

10. Fersht, A.R. Characterizing transition states in protein folding: an essential step in the puzzle. Curr. Opin. Struct. Biol. 1994, 5, 79-84.

11. Englander, S.W. Protein folding intermediates and pathways studied by hydrogen exchange. Annu. Rev. Biophys. Biomol. Struct. 2000, 29, 213-238.

12. Swanson, R.; Tsai, J. Pretty good guessing: protein structure prediction at CASP5. J. Bacteriol. 2003, 185, 3990-3993.

13. Schueler-Furman, O.; Wang, C.; Bradley, P.; Misura, K.; Baker, D. Progress in modeling of protein structures and interactions. Science 2005, 310, 638-642.

14. Kryshtafovych, A.; Fidelis, K.; Moult, J. CASP progress report. Proteins 2007, 69 (Suppl 8), 194-207.

15. Eaton, W.A.; Muñoz, V.; Hagen, S.J.; Jas, G.S.; Lapidus, L.J.; Henry, E.R.; Hofrichter, J. Fast kinetics and mechanisms in protein folding. Annu. Rev. Biophys. Biomol. Struct. 2000, 29, 327-359.

16. Maity, H.; Maity, M.; Krishna, M.M.G.; Mayne, L.; Englander, S.W. Protein folding: the stepwise assembly of foldon units. Proc. Natl. Acad. Sci. USA 2005, 102, 4741-4746.

17. Krantz, B.A.; Mayne, L.; Rumbley, J.; Englander, S.W.; Sosnick, T.R. Fast and slow intermediate accumulation and the initial barrier mechanism in protein folding. J. Mol. Biol. 2002, 324, 359-371. 
18. Bédard, S.; Krishna, M.M.; Mayne, L.; Englander, S.W. Protein folding: independent unrelated pathways or predetermined pathway with optional errors. Proc. Natl. Acad. Sci USA 2008, 105, $7182-7187$.

19. Dill, K.A.; Chan, H.S. From Levinthal to pathways to funnels. Nat. Struct. Biol. 1997, 4, 10-19.

20. Yon, J.M. Protein folding in the post-genomic era. J. Cell Mol. Med. 2002, 6, 307-327.

21. Gruebele, M. Downhill protein folding: evolution meets physics. C. R. Biol. 2005, 328, 701-712.

22. Vincent, J.J.; Tai, C.-H.; Sathyanarayana, B.K.; Lee, B. Assessment of CASP6 predictions for new and nearly new fold targets. Proteins 2005, 61 (Suppl 7), 67-83.

23. Jones, T.M.; Thirup, S. Using known substructures in protein model building and crystallography. EMBO J. 1986, 5, 819-822.

24. Unger, R.; Harel, D.; Wherland, S.; Sussman, J.L. A 3D building blocks approach to analyzing and predicting structures of proteins. Proteins 1989, 5, 355-373.

25. de Brevern, A.G.; Etchebest, C.; Hazout, S. Bayesian probabilistic approach for predicting backbone structures in terms of protein blocks. Proteins 2000, 41, 271-287.

26. Edgar, R.C.; Batzoglou, S. Multiple sequence alignment. Curr. Opin. Struct. Biol. 2006, 16, 368-373.

27. Cootes, A.P.; Curmi, P.M.G.; Cunningham, R.; Donnelly, C.; Torda, A.E. The dependence of amino acid pair correlations on structural environment. Proteins 1998, 32, 175-189.

28. White, S.H.; Jacobs, R.E. Statistical distribution of hydrophobic residues along the length of protein chains. Biophys. J. 1990, 57, 911-921.

29. Kauzmann, W. Some factors in the interpretation of protein denaturation. Adv. Protein Chem. 1959, 14, 1-63.

30. Rose, G.D.; Roy, S. Hydrophobic basis of packing in globular proteins. Proc. Natl. Acad. Sci. USA 1980, 77, 4643-4647.

31. Murphy, L.R.; Wallqvist, A.; Levy, R.M. Simplified amino acid alphabets for protein fold recognition and implications for folding. Protein Eng. 2000, 13, 149-152.

32. Etchebest, C.; Benros, C.; Bornot, A.; Camproux, A.-C.; de Brevern, A.G. A reduced amino acid alphabet for understanding and designing protein adaptation in mutation. Eur. Biophys. J. 2007, 36, 1059-1069.

33. Wang, J.; Wang, W. A computational approach to simplifying the protein folding alphabet. Nat. Struct. Biol. 1999, 6, 1033-1038.

34. Bacardit, J.; Stout, M.; Hirst, J.D; Valencia, A.; Smith, R.E.; Krasnogor, N. Automated alphabet reduction for protein datasets. BMC Bioinf. 2009, 10, 6 .

35. Vazquez, S.; Thomas, C.; Lew, R.A.; Humphreys, R.E. Favored and suppressed patterns on hydrophobic and nonhydrophobic amino acids in protein sequences. Proc. Natl. Acad. Sci. USA 1993, 90, 9100-9104.

36. Schwartz, R.; Istrail, S.; King, J. Frequencies of amino acid strings in globular protein sequences indicate suppression of blocks of consecutive hydrophobic residues. Protein Sci. 2001, 10, 1023-1031.

37. Schwartz, R.; King, J. Frequencies of hydrophobic and hydrophilic runs and alternations in proteins of known strcutre. Protein Sci. 2006, 15, 102-112. 
38. West, M.W.; Hecht, M.H. Binary patterning of polar and nonpolar amino acids in the sequences and structures of native proteins. Protein Sci. 1995, 4, 2032-2039.

39. Broome, B.M.; Hecht, M.H. Nature disfavors sequences of alternating polar and non-polar amino acids: implications for amyloidogenesis. J. Mol. Biol. 2000, 296, 961-968.

40. Xiong, H.; Buckwalter, B.L.; Shieh, H.M.; Hecht, M.H. Periodicity of polar and nonpolar amino acids is the major determinant of secondary structure in self-assembling oligomeric peptides. Proc. Natl. Acad. Sci. USA 1995, 92, 6349-6353.

41. Strait, B.J.; Dewey, T.G. The Shannon information entropy of protein sequences. Biophys. J. 1996, 71, 148-155.

42. Weiss, O.; Jiménez-Montaño, M.A.; Herzel, H. Information content of protein sequences. $J$. Theor. Biol. 2000, 206, 379-386.

43. Shannon, C.E. A mathematical theory of communication. The Bell System Tech. J. 1948, 27, 379-423, 623-656.

44. Chou, P.Y.; Fasman, G.D. Conformational parameters for amino acids in helical, $\beta$-sheet, and random coil regions calculated from proteins. Biochemistry 1974, 13, 211-222.

45. Richardson, J.S.; Richardson, D.C. Amino acid preferences for specific locations at the ends of $\alpha$ helices. Science 1988, 240, 1648-1652.

46. Aurora, R.; Rose, G.D. Helix capping. Protein Sci. 1998, 7, 21-38.

47. Gunasekaran, K.; Nagarajaram, H.A.; Ramakrishnan, C.; Balaram, P. Stereochemical punctuation marks in protein structures: glycine and proline containing helix stop signals. J. Mol. Biol. 1998, 275, 917-932.

48. Penel, S.; Hughes, E.; Doig, A.J. Side-chain structures in the first turn of the $\alpha$-helix. J. Mol. Biol. 1999, 287, 127-143.

49. Ermolenko, D.N.; Thomas, S.T.; Aurora, R.; Gronenborn, A.M; Makhatadze, G.I. Hydrophobic interactions at the Ccap position of the C-capping motif of $\alpha$-helices. J. Mol. Biol. 2002, 322, 123-135.

50. Pal, L.; Chakrabarti, P.; Basu, G. Sequence and structure patterns in proteins from an analysis of the shortest helices: implications for helix nucleation. J. Mol. Biol. 2003, 326, 273-291.

51. Wang, J.; Feng, J.-A. Exploring the sequence patterns in the $\alpha$-helices of proteins. Protein Eng. 2003, 16, 799-807.

52. Fonseca, N.A.; Camacho, R.; Magalhães, A.L. Amino acid pairing at the N- and C-termini of helical segments in proteins. Proteins 2008, 70, 188-196.

53. Lifson, S.; Sander, C. Antiparallel and parallel $\beta$-strands differ in amino acid residue preferences. Nature 1979, 282, 109-111.

54. Wouters, M.A.; Curmi, P.M.G. An analysis of side chain interactions and pair correlations within antiparallel $\beta$-sheets: the differences between backbone hydrogen-bonded and non-hydrogenbonded residue pairs. Proteins 1995, 22, 119-131.

55. Zhu, H.; Braun, W. Sequence specificity, statistical potentials, and three-dimensional structure prediction with self-correcting distance geometry calculations of $\beta$-sheet formation in proteins. Protein Sci. 1999, 8, 326-342.

56. Wilmot, C.M.; Thornton, J.M. Analysis and prediction of the different types of $\beta$-turn in proteins. J. Mol. Biol. 1988, 203, 221-232. 
57. Hutchinson, E.G.; Thornton, J.M. A revised set of potentials for \{beta $\}$-turn formation in proteins. Protein Sci. 1994, 3, 2207-2216.

58. Guruprasad, K.; Prasad, M.S.; Kumar, G.R. Analysis of $\gamma \beta, \beta \gamma, \gamma \gamma, \beta \beta$ multiple turns in proteins. J. Peptide Res. 2000, 56, 250-263.

59. Dasgupta, B.; Pal, L.; Basu, G.; Chakrabarti, P. Expanded turn conformations: characterization and sequence-structure correspondence in $\alpha$-turns, with implications in helix folding. Proteins 2004, 55, 305-315.

60. Colloc'h, N.; Cohen, F.E. $\beta$-breakers: an aperiodic secondary structure. J. Mol. Biol. 1991, 221, 603-613.

61. Chan, A.; Hutchinson, E.G.; Harris, D.; Thornton, J.M. Identification, classification, and analysis of beta-bulges in proteins. Protein Sci. 1993, 2, 1574-1590.

62. Daffner, C.; Chelvanayagam, G.; Argos, P. Structural characteristics and stabilizing principles of bent $\{$ beta\}-strands in protein tertiary architectures. Protein Sci. 1994, 3, 876-882.

63. Crasto, C.J.; Feng, J.-A. Sequence codes for extended conformation: a neighbor-dependent sequence analysis of loops in proteins. Proteins 2001, 42, 399-413.

64. Penel, S.; Morrison, R.G.; Mortishire-Smith, R.J.; Doig, A.J. Periodicity in $\alpha$-helix lengths and C-capping preferences. J. Mol. Biol. 1999, 293, 1211-1219.

65. Presta, L.G.; Rose, G.D. Helix signals in proteins. Science 1988, 240, 1632-1641.

66. Serrano, L.; Fersht, A.R. Capping and $\alpha$-helix stability. Nature 1989, 342, 296-299.

67. Serrano, L.; Sancho, J.; Hirshberg, M.; Ferscht, A.R. $\alpha$-helix stability in proteins. J. Mol. Biol. 1992, 227, 544-559.

68. Doig, A.J.; Baldwin, R.L. N- and C-capping preferences for all 20 amino acids in \{alpha $\}$-helical peptides. Protein Sci. 1995, 4, 1325-1335.

69. Sagermann, M.; Lars-Göran, M.; Baase, W.A.; Matthews, B.W. A test of proposed rules for helix capping: implications for protein design. Protein Sci. 2002, 11, 516-521.

70. Kapp, G.T.; Richardson, J.S.; Oas, T.G. Kinetic role of helix caps in protein folding is contextdependent. Biochemistry 2004, 43, 3814-3823.

71. Bang, D.; Gribenko, A.V.; Tereshko, V.; Kossiakoff, A.A.; Kent, S.B.; Makhatadze, G.I. Dissecting the energetics of protein $\alpha$-helix C-cap termination through chemical protein synthesis. Nat. Chem. Biol. 2006, 2, 139-143.

72. Doig, A.J. Recent advances in helix-coil theory. Biophys. Chem. 2002, 101-102, 281-293.

73. Wilson, C.L.; Boardman, P.E.; Doig, A.J.; Hubbard, S.J. Improved prediction for N-termini of $\alpha$ helices using empirical information. Proteins 2004, 57, 322-330.

74. Jiménez, M.A.; Muñoz, V.; Rico, M.; Serrano, L. Helix stop and start signals in peptides and proteins. The capping box does not necessarily prevent helix elongation. J. Mol. Biol. 1994, 242, 487-496.

75. Shortle, D. Propensities, probabilities, and the Boltzmann hypothesis. Protein Sci. 2003, 12, 1298-1302.

76. Klingler, T.M.; Brutlag, D.L. Discovering structural correlations in $\alpha$-helices. Protein Sci.1994, 3, 1847-1857.

77. Viguera, A.R.; Serrano, L. Side-chain interactions between sulfur-containing amino acids and phenylalanine in $\alpha$-helices. Biochemistry 1995, 34, 8771-8779. 
78. Walther, D.; Argos, P. Intrahelical side chain-side chain contacts: the consequences of restricted rotameric states and implications for helix engineering and design. Protein Eng. 1996, 9, 471-478.

79. Fernández-Recio, J.; Sancho, J. Intrahelical side chain interactions in $\alpha$-helices: poor correlation between energetics and frequency. FEBS Lett. 1998, 429, 99-103.

80. Andrew, C.D.; Penel, S.; Jones, G.R.; Doig, A.J. Stabilizing nonpolar/polar side-chain interactions in the $\alpha$-helix. Proteins 2001, 45, 449-455.

81. von Heijne, G.; Blomberg, C. The $\beta$ structure: inter-strand correlations. J. Mol. Biol. 1977, 117, 821-824.

82. Lifson, S. Specific recognition in the tertiary structure of $\beta$-sheets of proteins. J. Mol. Biol. 1980, 139, 627-639.

83. Hutchinson, E.G.; Sessions, R.B.; Thornton, J.M.; Woolfson, D.N. Determinants of strand register in antiparallel \{beta\}-sheets of proteins. Protein Sci. 1998, 7, 2287-2300.

84. Fooks, H.M.; Martin, A.C.R.; Woolfson, D.N.; Sessions, R.B.; Hutchinson, E.G. Amino acid pairing preferences in parallel $\beta$-sheets in proteins. J. Mol. Biol. 2006, 356, 32-44.

85. Mandel-Gutfreund, Y.; Zaremba, S.M.; Gregoret, L.M. Contributions of residue pairing to $\beta$ sheet formation: conservation and covariation of amino acid residue pairs on antiparallel $\beta$ strands. J. Mol. Biol. 2001, 305, 1145-1159.

86. Karplus, M; Weaver, D.L. Protein folding dynamics: the diffusion-collision model and experimental data. Protein Sci. 1994, 3, 650-668.

87. Wright, P.E.; Dyson, H.J. Intrinsically unstructured proteins: re-assessing the4 protein structurefunction paradigm. J. Mol. Biol. 1999, 293, 321-331.

88. Fink, A.L. Natively unfolded proteins. Curr. Opin. Struct. Biol. 2005, 15, 35-41.

89. Dunker, A.K.; Oldfield, C.J.; Meng, J.; Romero, P.; Yang, J.Y.; Chen, J.W.; Vacic, V.; Obradovic, Z.; Uversky, V.N. The unfoldomics decade: an update on intrinsically disordered proteins. BMC Genomics 2008, 9 (Suppl 2), S1.

90. Uversky, V.N.; Gillespie, J.R.; Fink, A.L. Why are "natively unfolded" proteins unstructured under physiologic conditions? Proteins 2000, 41, 415-427.

91. Romero, P; Obradovic, Z.; Li, X.; Garner, E.C.; Brown, C.J.; Dunker, A.K. Sequence complexity of disordered protein. Proteins 2001, 42, 38-48.

92. Dunker, A.K.; Lawson, J.D.; Brown, C.J.; Williams, R.M.; Romero, R.; Oh, J.S.; Oldfield, C.J.; Campen, A.M.; Ratliff, C.M.; Hipps, K.W.; Ausio, J.; Nissen, M.S.; Reeves, R.; Kang, C.; Kissinger, C.R.; Bailey, R.W.; Griswold, M.D.; Chiu, W.; Garner, E.C.; Obradovic, Z. Intrinsically disordered proteins. J. Mol. Graphics Modell. 2001, 19, 26-59.

93. Wetlaufer, D.B. Nucleation, rapid folding, and globular intrachain regions in proteins. Proc. Natl. Acad. Sci. USA 1973, 70, 697-701.

94. Rigden, D.J. Use of covariance analysis for the prediction of structural domain boundaries from multiple protein sequence alignments. Protein Eng. 2002, 15, 65-77.

95. Dong, Q.; Wang, X.; Lin, L.; Xu, Z. Domain boundary predictio nbased on profile domain linker propensity index. Comput. Biol. Chem. 2006, 30, 127-133.

96. Liu, J.; Rost, B. Sequence-based prediction nof protein domains. Nucleic Acids Res. 2004, 32, 3522-3530. 
97. Miyazaki, S.; Kuroda, Y.; Yokoyama, S. Identification of putative domain linkers by a neural network-application to a large sequence database. BMC Bioinf. 2006, 7, 323.

98. Ye, L.; Liu, T.; Wu, Z.; Zhou, R. Sequence-based protein domain boundary prediction using BP neural network with various property profiles. Proteins 2007, 71, 300-307.

99. Yoo, P.D.; Sikder, A.R.; Zhou, B.B.; Zomaya, A.Y. Improved general regression network for protein domain boundary prediction. BMC Bioinf. 2008, 9 (Suppl 1), 512

100. Wheelan, S.J.; Marchler-Bauer, A.; Bryant, S.H. Domain size distributions can predict domain boundaries. Bioinformatics 2000, 16, 613-618.

101. Suyama, M.; Ohara, O. DomCut: prediction of inter-domain linker regions in amino acid sequences. Bioinformatics 2003, 19, 673-674.

102. Galzitskaya, O.V.; Melnik, B.S. Prediction of protein domain boundaries from sequence alone. Protein Sci. 2003, 12, 696-701.

103. Levitt, M.; Chothia, C. Structural patterns in globular proteins. Nature 1976, 261, 552-558.

104. Chou, K.-C.; Zhang, C.-T. Prediction of protein structural classes. Crit. Rev. Biochem. Mol. Biol. 1995, 30, 275-349.

105. Nakashima, H.; Nishikawa, K.; Ooi, T. The folding type of a protein is relevant to the amino acid composition. J. Biochem. 1986, 99, 153-162.

106. Bu, W.-S.; Feng, Z.-P.; Zhang, Z.; Zhang, C.-T. Prediction of protein (domain) structural classes based on amino-acid index. Eur. J. Biochem. 1999, 266, 1043-1049.

107. Gu, F.; Chen, H.; Ni, J. Protein structural class prediction based on an improved statistical strategy. BMC Bioinf. 2008, 9 (Suppl 6), S5.

108. Luo, R.-Y.; Feng, Z.-P.; Liu, J.-K. Prediction of protein structural class by amino acid and polypeptide composition. Eur. J. Biochem. 2002, 269, 4219-4225.

109. Costantini, S.; Facchiano, A.M. Prediction of the protein structural class by specific peptide frequencies. Biochimie 2008, In press.

110. Mahalanobis, P.C. On the generalized distance in statistics. Proc. Natl. Inst. Sci. India 1936, 2, 49-55.

111. Ventura, S.; Serrano, L. Designing proteins from the inside out. Proteins 2004, 56, 1-10.

112. Santiveri, C.M.; Jiménez, M. A.; Rico, M.; Van Gunsteren, W.F.; Daura, X. $\beta$-hairpin folding and stability: molecular dynamics simulations of designed peptides in aqueous solution. J. Pept. Sci. 2004, 10, 546-65.

113. Muñoz, V.; Thompson, P.A.; Hofrichter, J; Eaton, W.A. Folding dynamics and mechanism of $\beta$ hairpin formation. Nature 1997, 390, 196-199.

114. Noguchi, T.; Matsuda, H.; Akiyama, Y. PDB-REPRDB: a database of representative protein chains from the Protein Data Bank (PDB). Nucleic Acids Res. 2001, 29, 219-220.

115. Kabsch, W.; Sander, C. Dictionary of protein secondary structure: pattern recognition of hydrogen-bonded and geometrical features. Biopolymers 1983, 22, 2577-2637.

116. Chiti, F.; Dobson, C.M. Amyloid formation by globular proteins under native conditions. Nat. Chem. Biol. 2009, 5, 15-22. 
117. Cohen, F.E.; Sternberg, M.J.E.; Taylor, W.R. Analysis and prediction of protein $\beta$-sheet structures by a combinatorial approach. Nature 1980, 285, 378-382.

(C) 2009 by the authors; licensee Molecular Diversity Preservation International, Basel, Switzerland. This article is an open-access article distributed under the terms and conditions of the Creative Commons Attribution license (http://creativecommons.org/licenses/by/3.0/). 